\title{
Neuroprotection against apoptosis of SK-N-MC cells using RMP-7- and lactoferrin-grafted liposomes carrying quercetin
}

This article was published in the following Dove Press journal:

International Journal of Nanomedicine

7 April 2017

Number of times this article has been viewed

\section{Yung-Chih Kuo \\ Chien-Wei Tsao}

Department of Chemical Engineering, National Chung Cheng University, Chia-Yi, Taiwan, Republic of China
Correspondence: Yung-Chih Kuo Department of Chemical Engineering, National Chung Cheng University, 168 University Rd., Min-Hsiung, Chia-Yi, Taiwan 62102, Republic of China Tel +8865272 04II ext33459

Fax +8865272 I206

Email chmyck@ccu.edu.tw
Abstract: A drug delivery system of quercetin (QU)-encapsulated liposomes (LS) grafted with RMP-7, a bradykinin analog, and lactoferrin (Lf) was developed to permeate the bloodbrain barrier (BBB) and rescue degenerated neurons, acting as an Alzheimer's disease (AD) pharmacotherapy. This colloidal formulation of QU-encapsulated LS grafted with RMP-7 and Lf (RMP-7-Lf-QU-LS) was used to traverse human brain microvascular endothelial cells (HBMECs) regulated by human astrocytes (HAs) and to treat SK-N-MC cells after an insult with cytotoxic $\beta$-amyloid (A $\beta$ ) fibrils. We found that surface RMP-7 and Lf enhanced the ability of QU to cross the BBB without inducing strong toxicity and damaging the tight junction. In addition, RMP-7-Lf-QU-LS significantly reduced $A \beta$-induced neurotoxicity and improved the viability of SK-N-MC cells. Compared with free QU, RMP-7-Lf-QU-LS could also significantly inhibit the expression of phosphorylated c-Jun N terminal kinase, phosphorylated p38, and phosphorylated tau protein at serine 202 by SK-N-MC cells, indicating an important role of RMP-7, Lf, and LS in protecting neurons against apoptosis. RMP-7-Lf-QU-LS is a promising carrier targeting the $\mathrm{BBB}$ to prevent $\mathrm{A} \beta$-insulted neurodegeneration and may have potential in managing $\mathrm{AD}$ in future clinical applications.

Keywords: Alzheimer's disease, blood-brain barrier, $\beta$-amyloid, drug targeting, neurodegeneration, pharmacotherapy

\section{Introduction}

Alzheimer's disease (AD) is one of the most serious neurodegenerative disorders in the world today. ${ }^{1}$ The most common symptom of AD is irreversible dementia, including chronic memory loss, episodic confusion about time and/or place, cognitive impairment, and personality change. ${ }^{2}$ AD patients also exhibited an atrophic change in brain parenchyma, arteriosclerotic tissue, and entanglement of neurofibrils in cerebral neurons. ${ }^{3}$ Molecular biology has shown that two major anatomical components responsible for $\mathrm{AD}$ pathology are deposited $\beta$-amyloid $(\mathrm{A} \beta)$ of abnormal senile plaques and hyperphosphorylated tau protein of cholinergic neurons in the hippocampus. ${ }^{4,5}$ In light of the pathological features, an apoptotic cell model to assist in understanding neurodegeneration would be of practical use for AD therapy. For example, cholinergic SK-N-MC cells with active neurochemical traits could be an eligible culture for this purpose because their $\alpha 7$ nicotinic acetylcholine receptors can recognize and internalize $\mathrm{A} \beta .^{6,7} \mathrm{~A}$ neuroprotective analysis of $\mathrm{A} \beta$-induced cytotoxicity also validated SK-N-MC cells as a useful system for AD-related study. ${ }^{8}$ In addition to the neuronal model, various drugs have been developed for pharmacotherapy in AD management. Quercetin (QU), for instance, had an antioxidative ability to eliminate free radicals 
and could ameliorate cognitive and memory dysfunction..$^{9-12}$ However, problems, including low solubility in the blood, rapid metabolism in the intestine and liver, and low uptake in the brain, have considerably restrained clinical application of QU. ${ }^{13}$ Hence, a modification with bioactive molecules to mediate the brain distribution of QU can be a possible approach to alleviating AD progression.

RMP-7, a bradykinin analog with a molecular weight of 1,096 Da, has higher selectivity in binding to the blood-brain barrier (BBB) than bradykinin and can stimulate the expression of $\mathrm{B}_{2}$ bradykinin receptors in the neural tissue. ${ }^{14}$ In fact, RMP-7 could facilitate the infiltration of pharmaceuticals into the central nervous system and might also regulate the tight junction opening of the BBB. ${ }^{15}$ In addition, liposomes (LS) associated with RMP-7 could accelerate delivery of Evans blue across the BBB. ${ }^{16}$ Lactoferrin (Lf), a member of the transferrin family, is a vital biomolecule in the blood that regulates immunological response, inflammation, and cell differentiation. ${ }^{17}$ Lf receptor (LfR) on human brain microvascular endothelial cells (HBMECs) activates receptormediated transcytosis for BBB permeation and controls neuronal physiology in the AD brain. ${ }^{18,19}$ It was reported that Lf could benefit the transport of anticancer medicine-loaded polymeric nanoparticles to brain tumor. ${ }^{20}$ Thus, RMP-7 and Lf may qualify as ingredients in pharmaceutical formulations for AD medication. Furthermore, the combined use of RMP-7 with Lf in brain targeting has never been reported. In a drug carrier system, RMP-7 and transferrin were used to improve the permeability of macromolecular nerve growth factor across the BBB. ${ }^{21}$ It is intriguing that the brain uptake of Lf was higher than that of transferrin, implying a higher efficiency of the former in permeating the BBB. ${ }^{22}$

The aim of this study was to develop QU-encapsulated LS grafted with RMP-7 and Lf (RMP-7-Lf-QU-LS) for use in neuroprotection in AD management. Since the bioavailability of QU in the brain is a critical issue in treating neurodegeneration, the influence of dual targeting on BBB permeability using RMP-7-Lf-QU-LS was investigated. RMP-7-grafted LS has been investigated previously; however, this study highlights the combination of RMP-7 and Lf on LS containing complicated lipid components for carrying and releasing QU. In addition, RMP-7-Lf-QU-LS was used to inhibit apoptotic progress and rescue neurons after an insult with $A \beta$, because $A \beta$ plaques attack neurons in the degenerated $\mathrm{AD}$ brain. We especially examined the effect of RMP-7-Lf-QU-LS on the viability of A $\beta$-insulted SK-N-MC cells and on the neuronal expression of phosphorylated c-Jun $\mathrm{N}$ terminal kinase (p-JNK), phosphorylated p38 (p-p38), and phosphorylated tau protein at serine 202 (p-S202).

\section{Materials and methods Materials}

1,2-Dipalmitoyl-sn-glycero-3-phosphocholine (DPPC), 1',3'-bis[1,2-dimyristoyl-sn-glycero-3-phospho]-snglycerol (cardiolipin [CL]), 1,2-distearoyl-sn-glycero-3phosphoethanolamine- $N$-[ carboxy(polyethylene glycol)2000] (DSPE-PEG(2000)-CA), and polycarbonate membrane were purchased from Avanti Polar Lipids (Alabaster, AL, USA). Soybean phosphatidylcholine (SPC), stearylamine (SA), cholesterol, fluorescein isothiocyanate (FITC)conjugated dextran 70000, Dulbecco's phosphate-buffered saline (DPBS), 2-( $N$-morpholino)ethanesulfonic acid (MES), 1-ethyl-3-(3-dimethylaminopropyl)carbodiimide (EDC), Lf, QuantiPro bicinchoninic acid (BCA) assay kit, phosphotungstic acid (PTA), sodium azide, propidium iodide (PI), formalin, 4',6-diamidino-2-phenylindole (DAPI), sodium dodecyl sulfate (SDS), and polyacrylamide gel (PAG) were obtained from Sigma-Aldrich (St Louis, MO, USA). HBMECs were purchased from Biocompare (South San Francisco, CA, USA). Human astrocytes (HAs), endothelial cell medium (ECM), and astrocyte medium were obtained from Sciencell (Carlsbad, CA, USA). SK-N-MC cells from human neuroblastoma were purchased from American Type Tissue Collection (Rockville, MD, USA). Anti-LfR monoclonal antibody, goat anti-mouse polyclonal secondary antibody immunoglobulin $\mathrm{G}$ (heavy and light) $(\operatorname{IgG}(\mathrm{H}+\mathrm{L}))$ conjugated with rhodamine, lysis buffer, anti-JNK (phospho T183 and Y185) (anti-p-JNK), goat anti-rabbit IgG (H + L) (horseradish peroxidase $[\mathrm{HRP}]$ ), and anti-A $\beta$ monoclonal antibody were obtained from Abcam (Cambridge, MA, USA). Sephadex G-100, polyvinylidene fluoride (PVDF), enhanced chemiluminescence (ECL), and select ECL were purchased from GE Healthcare (Piscataway, NJ, USA). Anti-p38 (phospho Thr180 + Tyr185) (anti-p-p38), anti-tau (phospho serine 202) (anti-p-S202), and anti-glyceraldehyde 3-phosphate dehydrogenase (GAPDH) were obtained from Cell Signaling (Danvers, MA, USA). Chloroform was purchased from JT Baker (Phillipsburg, NJ, USA), QU from Santa Cruz Biotechnology (Santa Cruz, CA, USA), methanol from Mallinckrodt Baker (Phillipsburg, NJ, USA), ultrapure water from Barnstead (Dubuque, IA, USA), $N$-hydroxysuccinimide (NHS) from Alfa Aesar (Ward Hill, MA, USA), RMP-7 from Alkermes (Cambridge, MA, USA), tris(hydroxymethyl) aminomethane (Tris) from Riedel-de Haën (Seelze, Germany), regenerated cellulose $(50 \mathrm{kDa})$ from Spectrum 
Laboratories (Rancho Dominguez, CA, USA), serum blocking solution from Zymed (South San Francisco, CA, USA), 2,3-bis-(2-methoxy-4-nitro-5-sulfophenyl)-2H-tetrazolium5-carboxanilide (XTT) from Biological Industries (Beit Haemek, Israel), anti-B ${ }_{2}$ bradykinin receptor antibody from Alomone (Jerusalem, Israel), Triton-X-100 from Acros (Geel, Belgium), aqueous mounting medium from Bio SB (Santa Barbara, CA, USA), A $\beta_{1-42}$ from Life Technologies (Carlsbad, CA, USA), Coomassie Blue Kit from Thermo Fisher Scientific (St Waltham, MA, USA), transfer buffer from Bio-Rad (Hercules, CA, USA), sample buffer from Millipore (Billerica, MA, USA), 96-well plate from Nalge Nunc (Rochester, NY, USA), 24-well flat-bottom tissue culture microtiter plate (polystyrene) from Midwest Scientific (St Louis, MO, USA), and microscope cover glass (gelatin coated) from Marienfeld GmbH (Lauda-Königshofen, Germany). Polyethylene terephthalate (PET) membrane (gelatin coated) and transwell were obtained from BD Falcon (Franklin Lakes, NJ, USA).

\section{Preparation of RMP-7-Lf-QU-LS}

\section{Preparation of QU-encapsulated LS (QU-LS)}

DPPC, SPC, CL, SA, cholesterol, and DSPE-PEG(2000)-CA were mixed in $1 \mathrm{~mL}$ of chloroform at $25^{\circ} \mathrm{C}$. In the typical case, the mole percentages of DPPC, SPC, CL, SA, cholesterol, and DSPE-PEG(2000)-CA were 25\%,17\%, 10\%, $5 \%, 40 \%$, and $3 \%$, respectively. When the mole percentage of SA varied, the content of DPPC, CL, cholesterol, and DSPE-PEG(2000)-CA remained constant and SPC balanced the variation at the level of SA. The weight percentage of QU was controlled at 4\% in the lipids. Fluorescent LS were obtained with the addition of $0.1 \%(\mathrm{w} / \mathrm{v})$ FITCconjugated dextran 70000 to the lipid phase. Chloroform was evaporated in a $50 \mathrm{~mL}$ round bottom flask using a rotary evaporator (Panchum, Kaohsiung, Taiwan) and a vacuum pump (Panchum) at $75 \mathrm{rpm}$ and $45^{\circ} \mathrm{C}$ for $15 \mathrm{~min}$. QU was dissolved in methanol. To prepare QU-LS, QU was added to the concentrated lipid film and evaporated again. A total of $2 \mathrm{mg} / \mathrm{mL}$ of the concentrated lipid film was solvated in DPBS at $45^{\circ} \mathrm{C}$, vibrated at $46 \mathrm{kHz}$ and $45^{\circ} \mathrm{C}$ for $30 \mathrm{~min}$, and compressed through a $100-\mathrm{nm}$ polycarbonate membrane in an extruder set (Avanti Polar Lipids) 15 times. Unloaded QU and QU-LS were separated using an upright column of $1.5 \times 50 \mathrm{~cm}$ packed with Sephadex G-100 at $25^{\circ} \mathrm{C}$. In all, $3 \mathrm{~mL}$ of the QU-LS suspension and $150 \mathrm{~mL}$ of DPBS were added to the column. A total of $2 \mathrm{~mL}$ of every effluent was collected, treated with $2 \mathrm{~mL}$ of methanol, analyzed using a high-performance liquid chromatograph (HPLC; JASCO,
Tokyo, Japan) with a reverse phase Hypersil BDS C18 column (Thermo Hypersil-Keystone, Bellefonte, PA, USA), and detected using an ultraviolet (UV) detector (UV-2075 Plus; JASCO) at $370 \mathrm{~nm}$. The mobile phase was 50\% (v/v) methanol in ultrapure water at a flow rate of $1 \mathrm{~mL} / \mathrm{min}$. The encapsulation efficiency of QU in QU-LS was defined as ([quantity of encapsulated QU in QU-LS]/[quantity of total QU in the preparation]) $\times 100 \%$.

\section{Grafting efficiency of Lf on QU-LS}

QU-LS were suspended in MES. The carboxyl groups on QU-LS were activated with $1 \mathrm{mM}$ of EDC and $1.5 \mathrm{mM}$ of $\mathrm{NHS}$ at $80 \mathrm{rpm}$ and $25^{\circ} \mathrm{C}$ for $1.5 \mathrm{~h}$. The suspension was mixed with $5-20 \mu \mathrm{g} / \mathrm{mL}$ of Lf at $80 \mathrm{rpm}$ and $25^{\circ} \mathrm{C}$ for $12 \mathrm{~h}$, and $3 \mathrm{~mL}$ of the suspension containing QU-LS grafted with Lf (Lf-QU-LS) and $150 \mathrm{~mL}$ of DPBS were added to the Sephadex G-100 column at $25^{\circ} \mathrm{C}$. Then, $2 \mathrm{~mL}$ of every effluent was collected. The quantity of unloaded Lf was evaluated with a QuantiPro BCA assay kit and determined using an enzyme-linked immunosorbent assay (ELISA) spectrophotometer (BioTek, Winooski, VT, USA) at $562 \mathrm{~nm}$. The grafting efficiency of Lf on QU-LS, $E_{\mathrm{Lf}}$, was defined as $E_{\mathrm{Lf}}(\%)=($ [quantity of total Lf in a preparation - quantity of unloaded Lf after gel chromatography]/[quantity of total Lf in a preparation]) $\times 100 \%$.

\section{Grafting efficiency of RMP-7 on Lf-QU-LS}

Activated Lf-QU-LS were mixed with 5-20 $\mu \mathrm{g} / \mathrm{mL}$ of RMP-7 at $80 \mathrm{rpm}$ and $25^{\circ} \mathrm{C}$ for $12 \mathrm{~h}$. The steps for grafting RMP-7 on Lf-QU-LS using EDC and NHS were analogous to those for grafting Lf on QU-LS, which is described in the "Grafting efficiency of Lf on QU-LS" section. A total of $3 \mathrm{~mL}$ of the suspension containing Lf-QU-LS grafted with RMP-7, defined as RMP-7-Lf-QU-LS, and $150 \mathrm{~mL}$ of DPBS were added to the Sephadex G-100 column at $25^{\circ} \mathrm{C}$. The grafting efficiency of RMP-7 on Lf-QU-LS, $E_{\text {RMP-7 }}$, was defined as $E_{\mathrm{RMP}-7}(\%)=([$ quantity of total RMP-7 in a preparation quantity of unloaded RMP-7 after gel chromatography] [quantity of total RMP-7 in a preparation]) $\times 100 \%$.

\section{Characterization of RMP-7-Lf-QU-LS}

\section{Particle size and charge}

The cumulant $Z$-averaged diameter, $D$, and zeta potential, $\zeta$, of RMP-7-Lf-QU-LS were analyzed using a Zetasizer 3000 HSA with a photon correlation spectroscope and a laser Doppler velocimeter (Malvern, Worcestershire, UK) at $25^{\circ} \mathrm{C}$. In all, $3 \mathrm{~mL}$ of $1 \mathrm{mg} / \mathrm{mL}$ of RMP-7-Lf-QU-LS in Tris buffer at $\mathrm{pH} 7.4$ was used in every study. 
Scanning electron microscope (SEM) and transmission electron microscope (TEM) morphology

The geometry of RMP-7-Lf-QU-LS was investigated using a field emission scanning electron microscope (FESEM; JSM-6330 TF; JEOL, Tokyo, Japan). In all, $10 \mu \mathrm{L}$ of $2 \mathrm{mg} / \mathrm{mL}$ of RMP-7-Lf-QU-LS in Tris buffer was ultrasonically vibrated for $1 \mathrm{~min}$, dropped on a cover slide, dried at $25^{\circ} \mathrm{C}$ for $24 \mathrm{~h}$, vacuum dried, glued with carbon paint, and sputter coated with platinum. The structure of RMP-7-Lf-QU-LS was examined using a TEM (JEM-1400; JEOL). In all, $10 \mu \mathrm{L}$ of $2 \mathrm{mg} / \mathrm{mL}$ of RMP-7-Lf-QU-LS in Tris buffer was loaded on a carbon-coated 200-mesh copper grid for $1 \mathrm{~min}$ and were treated with $10 \mu \mathrm{L}$ of $2 \%(\mathrm{w} / \mathrm{v})$ PTA solution for $2 \mathrm{~min}$.

\section{X-ray photoelectron spectroscope (XPS) spectra}

Surface atoms on RMP-7-Lf-QU-LS were analyzed using an XPS (Kratos, Kanagawa, Japan) under a light beam area of $300 \times 700 \mathrm{~mm}$ at $2 \times 10^{-7} \mathrm{~Pa}$ and $300 \mathrm{~W}$. A total of $2 \mathrm{mg} / \mathrm{mL}$ of RMP-7-Lf-QU-LS was dropped on a coverslip of $5 \times 5 \mathrm{~mm}$, dehydrated, and vacuum dried for $15 \mathrm{~min}$.

\section{Release kinetics of QU}

In all, $1.5 \mathrm{mg} / \mathrm{mL}$ of RMP-7-Lf-QU-LS in DPBS containing $0.05 \%(\mathrm{w} / \mathrm{v})$ sodium azide at $\mathrm{pH} 7.4$ was used for the dissolution study. A dialysis tube of regenerated cellulose was trimmed to $3.3 \times 20 \mathrm{~cm}$. A total of $10 \mathrm{~mL}$ of the suspension was added to the dialysis tube in a $100 \mathrm{~mL}$ flask containing $50 \mathrm{~mL}$ of DPBS at $\mathrm{pH} 7.4$ and was shaken in a bath reciprocal shaker at $50 \mathrm{rpm}$ and $37^{\circ} \mathrm{C}$ for $48 \mathrm{~h}$. In all, $100 \mu \mathrm{L}$ of dialyzate was taken out from the flask at a specific time point and the quantity of QU was evaluated using an HPLC-UV at $370 \mathrm{~nm}$. The dialyzate was compensated with $100 \mu \mathrm{L}$ of fresh DPBS immediately after sampling. The cumulative percentage of QU released from RMP-7-Lf-QU-LS, $P_{\mathrm{QU}}$, was defined as ([quantity of cumulative QU in DPBS]/[quantity of encapsulated QU in RMP-7-Lf-QU-LS]) $\times 100 \%$.

\section{Transport across the BBB using RMP-7-Lf-QU-LS}

\section{Toxicity to HBMECs and HAs}

The methods used to cultivate HBMECs and HAs have been described previously. ${ }^{23}$ HBMECs at a density of $7.5 \times 10^{3}$ cells/well in gelatin-pretreated 96-well plates were cultured with $150 \mu \mathrm{L}$ of endothelial cell medium per well in a humidified $\mathrm{CO}_{2}$ incubator (NuAire, Plymouth, $\mathrm{MN}$, USA) at $37^{\circ} \mathrm{C}$ for $8 \mathrm{~h}$ and incubated with $0.025 \%(\mathrm{w} / \mathrm{v})$
RMP-7-Lf-QU-LS for $12 \mathrm{~h}$. The viability of HBMECs was assayed with XTT and was evaluated using an ELISA spectrophotometer at $450 \mathrm{~nm}$. Cultured HBMECs were reacted with $50 \mu \mathrm{L}$ of XTT per well containing $2 \%(\mathrm{v} / \mathrm{v})$ activation solution in a humidified $\mathrm{CO}_{2}$ incubator for $4 \mathrm{~h}$. Analogous procedures were used to determine the viability of HAs cultured with the astrocyte medium. The viability of BBB cells (HBMECs and HAs), $P_{\mathrm{V}, \mathrm{BBB}}$, was defined as $P_{\mathrm{V}, \mathrm{BBB}}(\%)=([$ optical density of HBMECs or HAs after treatment with RMP-7-Lf-QU-LS - optical density of XTT]/ [optical density of HBMECs or HAs - optical density of $\mathrm{XTT}]) \times 100 \%$.

\section{Transendothelial electrical resistance (TEER) of the} BBB after treatment with RMP-7-Lf-QU-LS

The method used to establish HBMECs regulated by HAs (HBMEC/HA) on a PET membrane with a pore size of $0.4 \mu \mathrm{m}$ and a surface area of $0.3 \mathrm{~cm}^{2}$ in a transwell was described in our previous study. ${ }^{24}$ In all, $0.025 \%$ (w/v) RMP-7-Lf-QU-LS was added to the upper donor chamber facing the HBMECs, cultured in ECM in a 24-well flatbottom tissue culture microtiter plate, and placed in a humidified $\mathrm{CO}_{2}$ incubator for $5 \mathrm{~h}$. The donor chamber and lower receiver chamber facing the HAs were filled with a fresh medium before determining the electrical resistance of the membrane using a Millicell electrical resistance system (Millipore). The TEER of HBMEC/HA was defined as TEER $\left(\Omega \cdot \mathrm{cm}^{2}\right)=($ electrical resistance of membrane with HBMEC/ HA - electrical resistance of original membrane without cells) $\times$ surface area of the membrane.

\section{Ability of PI to cross the BBB after treatment with RMP-7-Lf-QU-LS}

After treatment with RMP-7-Lf-QU-LS, the medium of the donor chamber was replaced with a fresh medium containing $0.25 \mathrm{mg} / \mathrm{mL}$ of PI and cultured in a $\mathrm{CO}_{2}$ incubator for $5 \mathrm{~h}$. The quantity of PI in the receiver chamber was determined using a microplate fluorescent reader (Synergy HT; BioTek) with excitation at $485 \mathrm{~nm}$ and emission at $590 \mathrm{~nm}$. The ability of PI to cross the BBB, $P_{\mathrm{BBB}, \mathrm{PP}}$, was defined as ([ability of PI to cross the membrane with $\mathrm{HBMEC} / \mathrm{HA}]^{-1}-$ [ability of PI to cross the membrane without cells $\left.]^{-1}\right)^{-1}$.

\section{Ability of QU to cross the BBB using RMP-7-Lf-QU-LS}

A total of $0.025 \%(w / v)$ RMP-7-Lf-QU-LS was added to the donor chamber and cultured in a humidified $\mathrm{CO}_{2}$ incubator for $5 \mathrm{~h}$. In all, $20 \mu \mathrm{L}$ of the liquid in the receiver 
chamber was sampled every $2.5 \mathrm{~h}$, treated with methanol at $37^{\circ} \mathrm{C}$ for $30 \mathrm{~min}$, and analyzed using the HPLC-UV system (at $370 \mathrm{~nm}$ ) described in the "Preparation of QU-LS" section. A total of $20 \mu \mathrm{L}$ of the fresh medium was added to the receiver chamber immediately. The ability of QU to cross the BBB, $P_{\mathrm{BBB}, \mathrm{QU}}$, was defined as ([ability of QU to cross the membrane with $\mathrm{HBMEC} / \mathrm{HA}]^{-1}-[$ ability of QU to cross the membrane without cells $\left.]^{-1}\right)^{-1}$.

\section{Immunochemical staining of $L f R$ and $B_{2}$ bradykinin receptor in HBMECs during interaction with RMP-7-Lf-QU-LS}

HBMECs at a density of $1 \times 10^{5}$ cells $/ \mathrm{cm}^{2}$ were seeded on a microscope cover glass in a 24 -well plate, cultured in a humidified $\mathrm{CO}_{2}$ incubator for $8 \mathrm{~h}$, treated with $0.025 \%$ (w/v) RMP-7-Lf-QU-LS, and cultured in a humidified $\mathrm{CO}_{2}$ incubator for $3 \mathrm{~h}$. In preparing the staining sample, cultured HBMECs were fixed with $10 \%(\mathrm{v} / \mathrm{v})$ formalin at $25^{\circ} \mathrm{C}$ for $10 \mathrm{~min}$, treated with $0.5 \%(\mathrm{v} / \mathrm{v})$ Triton-X-100 at $25^{\circ} \mathrm{C}$ for $10 \mathrm{~min}$, and incubated with serum blocking solution at $25^{\circ} \mathrm{C}$ for $30 \mathrm{~min}$. The sample was reacted with $1 \mu \mathrm{g} / \mathrm{mL}$ of antiLfR monoclonal antibody and/or with anti-B $\mathrm{B}_{2}$ bradykinin receptor antibody $(1: 1,000)$ at $4^{\circ} \mathrm{C}$ for $12 \mathrm{~h}$, stained with goat anti-mouse polyclonal secondary antibody $\mathrm{IgG}$ $(\mathrm{H}+\mathrm{L})$ conjugated with rhodamine $(1: 100)$ at $25^{\circ} \mathrm{C}$ for $1 \mathrm{~h}$ in darkness, treated with $0.5 \%(\mathrm{w} / \mathrm{v})$ DAPI in $0.1 \%(\mathrm{v} / \mathrm{v})$ Triton-X-100 at $25^{\circ} \mathrm{C}$ for $3 \mathrm{~min}$ in darkness, and incubated in an aqueous mounting medium. RMP-7-Lf-QU-LS, LfR and/or $\mathrm{B}_{2}$ bradykinin receptor, and HBMECs were visualized using a confocal laser scanning microscope (LSM 510; Zeiss, Oberkochen, Germany) with excitation at $350 \mathrm{~nm}$ (nuclei, blue), $490 \mathrm{~nm}$ (RMP-7-Lf-QU-LS, green), and $555 \mathrm{~nm}$ (LfR and/or $\mathrm{B}_{2}$ bradykinin receptor, red) and emission at $475 \mathrm{~nm}$, $520 \mathrm{~nm}$, and $565 \mathrm{~nm}$.

\section{Rescue of degenerated SK-N-MC cells from apoptosis using RMP-7-Lf-QU-LS Viability of SK-N-MC cells after treatment with RMP-7-Lf-QU-LS}

The methods used to culture SK-N-MC cells and prepare fibrillar $\mathrm{A} \beta_{1-42}$ were described in our previous study. ${ }^{25}$ For the viability assay, the steps used to treat SK-N-MC cells were analogous to those for HBMECs and HAs described in the "Toxicity to HBMECs and HAs" section, except for the addition of $15 \mu \mathrm{M}$ of fibrillar $A \beta_{1-42}$ to the culture medium for SK-N-MC cells. The viability of SK-N-MC cells, $P_{\mathrm{V}, \mathrm{SK}-\mathrm{N}-\mathrm{MC}}$ was defined as $P_{\mathrm{V}, \mathrm{SK}-\mathrm{N}-\mathrm{MC}}(\%)=([$ optical density of SK-N-MC cells after treatment with RMP-7-Lf-QU-LS - optical density of XTT]/[optical density of SK-N-MC cells - optical density of XTT]) $\times 100 \%$.

Western blot of p-p38 and p-S202 after treatment with RMP-7-Lf-QU-LS

SK-N-MC cells at a density of $1 \times 10^{5}$ cells/well were seeded in a 12 -well plate, treated with $15 \mu \mathrm{M}$ of fibrillar $A \beta_{1-42}$ and RMP-7-Lf-QU-LS, and lyzed with $200 \mu \mathrm{L}$ of lysis buffer per well for $30 \mathrm{~min}$. A total of $2 \mu \mathrm{L}$ of decomposed protein solution from SK-N-MC cells was diluted with $198 \mu \mathrm{L}$ of ultrapure water, and $150 \mu \mathrm{L}$ of diluted solution was reacted with $150 \mu \mathrm{L}$ of Coomassie Blue Kit in a 96-well plate. The protein content in the sample was determined using an ELISA spectrophotometer at $595 \mathrm{~nm}$. The sample was heated to $95^{\circ} \mathrm{C}$ and added to a $25 \%$ (v/v) sample buffer containing $25 \mu \mathrm{g}$ of protein per vial. In the SDS-PAG electrophoresis, proteins were segregated in separating gel at $60 \mathrm{~V}$ for $15 \mathrm{~min}$ and then at $120 \mathrm{~V}$ for $30 \mathrm{~min}$; relocated to the PVDF membrane in transfer buffer at $400 \mathrm{~mA}$ and $4^{\circ} \mathrm{C}$ for $90 \mathrm{~min}$; incubated with $5 \%(\mathrm{w} / \mathrm{v})$ skim milk for $1 \mathrm{~h}$; reacted with anti-p-JNK (1:1,000), anti-p-p38 (1:1,000), anti-p-S202 (1:1,000), and anti-GAPDH $(1: 1,000)$ at $4^{\circ} \mathrm{C}$ for $12 \mathrm{~h}$; and stained with goat anti-rabbit $\operatorname{IgG}(\mathrm{H}+\mathrm{L})$ (HRP; $1: 2,000)$ at $25^{\circ} \mathrm{C}$ for $1.5 \mathrm{~h}$. Anti-GAPDH was augmented with ECL, and anti-p-JNK, anti-p-p38, and anti-p-S202 were augmented with select ECL for $1 \mathrm{~min}$. Protein bands in lanes were visualized using a Luminescence Image Analysis System (Fujifilm, Tokyo, Japan), and the relative quantity of protein was analyzed using the ImageJ software (version 1.50; National Institutes of Health, Bethesda, MD, USA).

Immunochemical staining of RMP-7-Lf-QU-LS adjacent to $A \beta$-insulted SK-N-MC cells

The steps taken to prepare the staining sample of SK$\mathrm{N}-\mathrm{MC}$ cells were analogous to those of HBMECs described in the "Immunochemical staining of LfR and $\mathrm{B}_{2}$ bradykinin receptor in HBMECs during interaction with RMP-7-LfQU-LS" section. Differences between the two systems were the addition of $15 \mu \mathrm{M}$ of fibrillar $\mathrm{A} \beta_{1-42}$ with RMP7-Lf-QU-LS and the replacement of anti-LfR and anti-B bradykinin receptor antibody with anti-A $\beta$ monoclonal antibody (1:400). The procedure for fluorescent imaging of SK-N-MC cells was the same as that for HBMECs.

\section{Statistics}

Data are presented as mean \pm standard deviation. Statistical comparison between groups was performed using a one-way analysis of variance followed by Tukey's honest significant difference test. 


\section{Results and discussion Colloidal delivery system of RMP-7-Lf-QU-LS}

Figure 1A shows the average diameter and zeta potential of RMP-7-Lf-QU-LS. The insets in this figure reveal the corresponding SEM morphology at mole percentage of SA in the lipid bilayer $\left(r_{\mathrm{SA}}\right)=5 \%, 15 \%$, and $25 \%$. As seen in Figure 1A, an increase in the mole percentage of SA from 5\% to $25 \%$ enlarged the particles and increased the zeta potential. This was because SA was positively charged in the bilayer and could increase repulsion between positively charged groups in the membrane, leading to a random liposomal structure and large particles. The particle size in the SEM image was consistent with the data. This SEM morphology was similar to that reported in the literature on LS comprising phospholipids and cholesterol. ${ }^{26}$ In this study, the encapsulation efficiency of QU in QU-LS was $52.61 \% \pm 3.27 \%$ at $r_{\mathrm{SA}}=5 \%(\mathrm{n}=3)$. QU in methanol was added to and incorporated in a lipid thin film via hydrophobic affinity. It was reported that hydrophilic and hydrophobic drugs could be entrapped in LS. ${ }^{27}$ Figure 1B reveals the coated structure of RMP-7 and/or Lf on the particles. The bilayer structure of QU-LS is shown in Figure 1Ba. Figure 1Bb exhibits a dark contour of modified Lf. RMP-7-Lf-QU-LS reveals prominent negative stains in Figure 1Bc. Figure $1 \mathrm{C}$ shows the grafting efficiency of RMP-7 and Lf. As seen in this figure, an increase in the mole percentage of SA from $5 \%$ to $25 \%$ reduced the grafting efficiency of RMP-7 and Lf, in general. This was because positive charges on the particles repelled positively charged RMP-7 and Lf in the grafting medium. ${ }^{28}$ Figure 1D shows the signals of surface atoms on RMP-7-Lf-QU-LS. In this figure, an increase in the mole percentage of SA from $0 \%$ to $5 \%$ enhanced the quantity of surface nitrogen at $397 \mathrm{eV}$. This was because the atomic ratio of nitrogen to all atoms in SA was higher than that in QU-LS without SA. In addition, RMP-7 and Lf had abundant nitrogen atoms and significantly promoted the nitrogen content on QU-LS. Figure 1E shows the release kinetics of QU from RMP-7Lf-QU-LS. The initial leakage of encapsulated QU from the current LS preparation was negligible. The release rate was in the order of RMP-7-Lf-QU-LS < Lf-QU-LS < QU-LS for $48 \mathrm{~h}$, suggesting the sustained release characteristics of RMP-7-Lf-QU-LS. This was because surface RMP-7 and/or Lf shielded the particle surface, obstructed the diffusion path, increased the migration distance to the medium, and retarded the dissolution of QU from the bilayer. As a result, QU could migrate and diffuse to DPBS. Previous studies reported that the release kinetics of QU to aqueous medium could be biphasic, including burst and prolonged stages; this was also observed in our study. ${ }^{29-31}$

\section{Delivery of QU across the BBB using RMP-7-Lf-QU-LS}

Figure 2A shows the cytotoxicity of RMP-7-Lf-QU-LS to HBMECs and HAs. As seen in this figure, the viability of the BBB cells was high, suggesting that RMP-7-Lf-QU-LS could be appropriate drug delivery carriers for targeting without seriously imperiling the BBB, although LS might induce inflammatory responses. ${ }^{32}$ Figure $2 \mathrm{~A}$ also indicates that the viability of HBMECs and HAs was comparable. Figure $2 \mathrm{~B}$ shows the TEER of the BBB and the ability of PI to cross the BBB after treatment with RMP-7-Lf-QU-LS. A high TEER and a low ability of PI to permeate the BBB represent a compact tight junction. As indicated in Figure 2B, an increase in the concentration of RMP-7 and Lf slightly decreased the TEER and increased the ability of PI to cross the BBB. This suggested that RMP-7-Lf-QU-LS might slightly enhance paracellular drug delivery. ${ }^{33}$ It has been reported that LS could not severely damage the integrity of the BBB. ${ }^{34}$ Figure $2 \mathrm{C}$ shows the ability of QU to cross the BBB using RMP-7-Lf-QU-LS. This figure demonstrates the enhancement of the ability of QU to cross the BBB by increasing the level of surface RMP- 7 and Lf. The ability of free QU to cross the BBB was $6.42 \pm 0.51 \mathrm{~cm} / \mathrm{s}(\mathrm{n}=3)$, which was greater than the ability of QU using Lf-QU-LS prepared at $5 \mu \mathrm{g} / \mathrm{mL}$ of Lf. This was because hydrophobic drugs such as QU with a molecular weight of 302.24 Da might be easier to transport across the BBB. ${ }^{35}$ However, QU loaded in nanocarriers such as RMP-7-Lf-QU-LS could have controlled release characteristics and targeting ability to rescue neurons in the brain. In addition, the ability of QU to cross the BBB using QU-encapsulated LS grafted with RMP-7 (RMP-7-QU-LS) that was prepared at $5 \mu \mathrm{g} / \mathrm{mL}$ of RMP-7 was $5.76 \pm 1.87 \mathrm{~cm} / \mathrm{s}$ $(\mathrm{n}=3)$, which was lower than that for free QU. However, the ability of QU to cross the BBB using RMP-7-QU-LS prepared at $10 \mu \mathrm{g} / \mathrm{mL}$ of RMP-7 increased to $7.03 \pm 1.45 \mathrm{~cm} / \mathrm{s}(\mathrm{n}=3)$, which was comparable to that for free QU. Figure $2 \mathrm{C}$ shows that RMP-7-Lf-QU-LS prepared at $10 \mu \mathrm{g} / \mathrm{mL}$ of $\mathrm{Lf}$ and $10 \mu \mathrm{g} / \mathrm{mL}$ of RMP-7 could double the ability of free QU to cross the BBB and significantly increased the ability of QU when using Lf-QU-LS alone. A study on chemotherapy for brain cancer indicated that a modification of targeting molecules was a potent strategy to deliver daunorubicin across the BBB. ${ }^{36}$ Figure 2D shows immunocytochemical staining images unveiling the internalization of RMP-7-Lf-QU-LS by HBMECs through recognition of $\mathrm{B}_{2}$ bradykinin receptor 

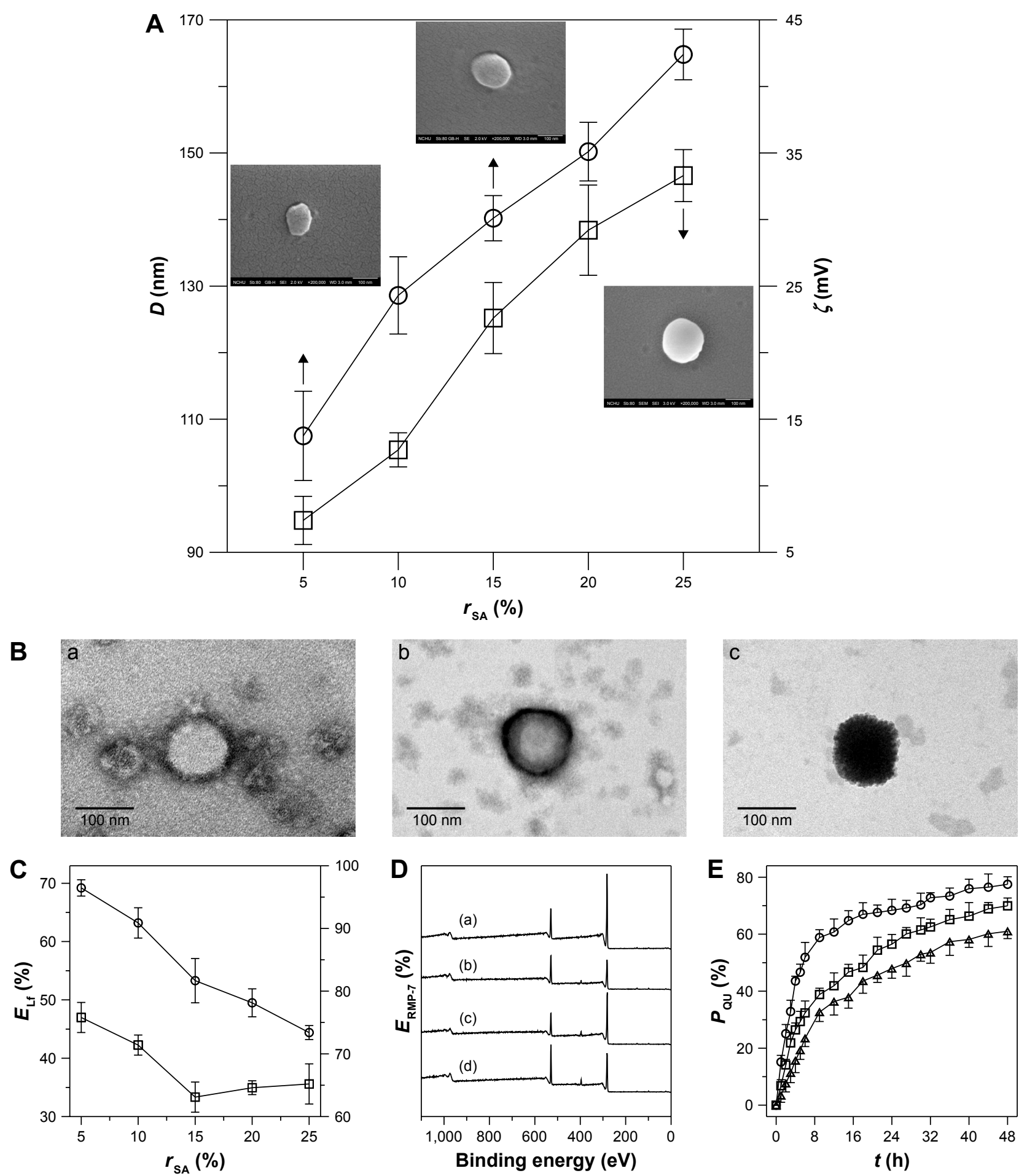

Figure I Physicochemical properties of RMP-7-Lf-QU-LS as a colloidal delivery system.

Notes: (A) Effect of SA on average particle diameter and zeta potential $\left(C_{\mathrm{RMP}-7}=20 \mu \mathrm{g} / \mathrm{mL} ; C_{\mathrm{Lf}}=20 \mu \mathrm{g} / \mathrm{mL} ; \bigcirc\right.$, average particle diameter [n=3]; $\square$, zeta potential [n=3]; inset: $S E M$ images of particles at $r_{S A}=5 \%, r_{S A}=15 \%$, and $r_{S A}=25 \%$ ); particle diameter and zeta potential increased with increases in the SA level. (B) Effect of RMP-7 and Lf on TEM image ( $\left.r_{S A}=5 \%\right)$ : (a) QU-LS, (b) Lf-QU-LS ( $\left.C_{\mathrm{Lf}}=20 \mu \mathrm{g} / \mathrm{mL}\right)$, and (c) RMP-7-Lf-QU-LS $\left(C_{\mathrm{RMP}-7}=20 \mu \mathrm{g} / \mathrm{mL} ; C_{\mathrm{Lf}}=20 \mu \mathrm{g} / \mathrm{mL}\right)$; stronger black stains of particles with grafted RMP-7 and Lf. (C) Effect of SA on grafting efficiency $\left(\bigcirc\right.$, grafting efficiency of Lf on QU-LS [n=3]; $\square$, grafting efficiency of RMP-7 on Lf-QU-LS [ $\left.\left.C_{L f}=20 \mu g / m L ; n=3\right]\right)$; grafting efficiency generally decreased with increases in the SA level. (D) XPS spectra: (a) QU-LS ( $r_{\mathrm{SA}}=0 \%$ ), (b) QU-LS $\left(r_{\mathrm{SA}}=5 \%\right.$ ), (c) Lf-QU-LS $\left(r_{\mathrm{SA}}=5 \% ; C_{\mathrm{Lf}}=20 \mu \mathrm{g} / \mathrm{mL}\right),(\mathrm{d}) \mathrm{RMP}-7-\mathrm{Lf}-\mathrm{QU}-\mathrm{LS}\left(r_{\mathrm{SA}}=5 \% ; C_{\mathrm{RMP}-7}=20 \mu g / \mathrm{mL} ; C_{\mathrm{Lf}}=20 \mu \mathrm{g} / \mathrm{mL}\right)$; quantity of surface nitrogen increased with increases in the SA, RMP-7, and Lf levels because SA, RMP-7, and Lf possessed more nitrogen than other components in QU-LS. (E) Effect of RMP-7 and Lf on dissolution of QU ( $r_{\mathrm{SA}}=5 \%$;, QU-LS; $\square$, Lf-QU-LS [ $\left.C_{\mathrm{Lf}}=20 \mu \mathrm{g} / \mathrm{mL}\right] ; \Delta$, RMP-7-Lf-QU-LS [ $\left.\left.C_{\mathrm{RMP}-7}=20 \mu \mathrm{g} / \mathrm{mL} ; C_{\mathrm{Lf}}=20 \mu \mathrm{g} / \mathrm{mL} ; \mathrm{n}=3\right]\right)$; slow release rate of QU suggested the sustained release characteristics of RMP-7-Lf-QU-LS. $C_{\mathrm{RMP}-7}$, concentration of RMP-7; $C_{\mathrm{Lf}}$, concentration of Lf; $r_{\mathrm{SA}}$, mole percentage of SA in the lipid bilayer. $E_{L \mathrm{f}}$, grafting efficiency of lactoferrin on QU-LS (\%); $E_{\text {RMP-7 }}$, grafting efficiency of RMP-7 on Lf-QU-LS (\%); $P_{\text {OU }}$, ability of quercetin to cross the BBB using RMP-7-Lf-QU-LS (cm/s).

Abbreviations: RMP-7-Lf-QU-LS, quercetin-encapsulated liposomes grafted with RMP-7 and lactoferrin; SA, stearylamine; SEM, scanning electron microscope; Lf, lactoferrin; TEM, transmission electron microscope; QU-LS, quercetin-encapsulated liposomes; Lf-QU-LS, quercetin-liposomes grafted with lactoferrin; XPS, X-ray photoelectron spectroscope; QU, quercetin; BBB, blood-brain barrier. 
and LfR. Ample LfRs on HBMECs appear in Figure 2Db, and ample $B_{2}$ bradykinin receptors and LfRs on HBMECs are seen in Figure 2Da, c, and d. Moreover, the green intensity was in the order of QU-LS $<$ Lf-QU-LS $<$ RMP-7-Lf-QU-LS, and a high concentration of RMP-7 could promote the uptake of RMP-7-Lf-QU-LS. This suggested that surface RMP-7 and Lf could target HBMECs and favor the delivery of QU across the $\mathrm{BBB}$ and that the images in Figure 2D were consistent with the data in Figure $2 \mathrm{C}$.

\section{Rescue of degenerated SK-N-MC cells using RMP-7-Lf-QU-LS}

Figure $3 \mathrm{~A}$ shows the viability of $\mathrm{A} \beta$-insulted SK-N-MC cells after salvage with RMP-7-Lf-QU-LS. As seen in this figure,
$\mathrm{A} \beta$ induced strong neurotoxicity and RMP-7-Lf-QU-LS could protect SK-N-MC cells from significant neurodegeneration. QU-LS containing CL in bilayers could also reduce apoptosis of SK-N-MC cells. This was because of the affinity of $\mathrm{CL}$ to $\mathrm{A} \beta .{ }^{37} \mathrm{~A}$ reduced toxicity to SK-N-MC cells has also been reported due to the high affinity of AA3E2 to $\mathrm{A} \beta .{ }^{38}$ In addition, $\mathrm{CL}$ could form a complex for transporting electrons by conjugating a portion of MGST1 antibody. ${ }^{39}$ Figure 3A reveals that RMP-7-Lf-QU-LS could also enhance the viability of SK-N-MC cells via a targeting effect of Lf. Figure 3B shows the expression of p-JNK, p-p38, and p-S202 in $\mathrm{A} \beta$-insulted SK-N-MC cells after salvage with RMP-7Lf-QU-LS. This figure indicates that $\mathrm{A} \beta$ induced substantial expressions of p-JNK, p-p38, and p-S202 in SK-N-MC cells.
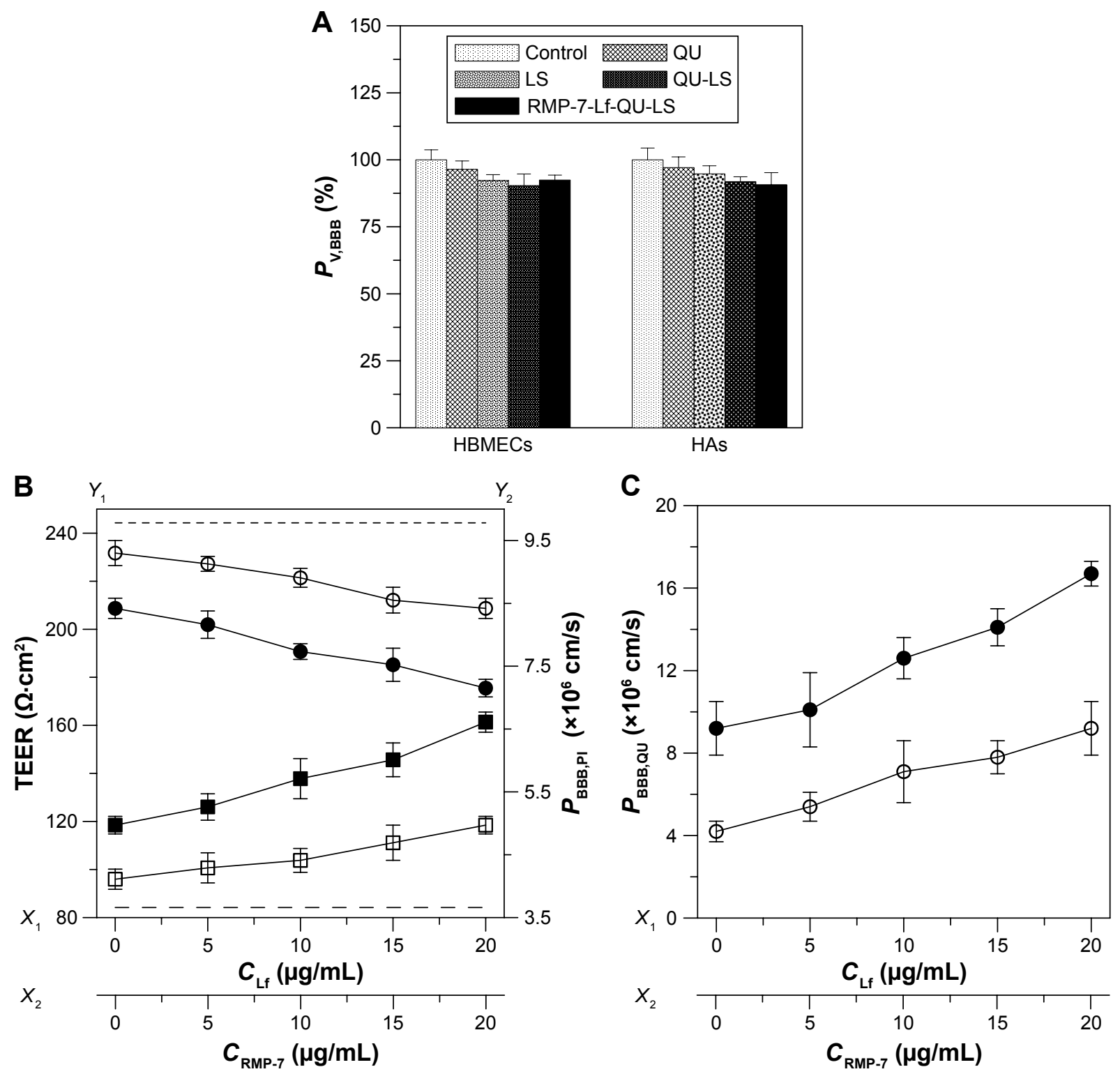

Figure 2 (Continued) 


\section{D}
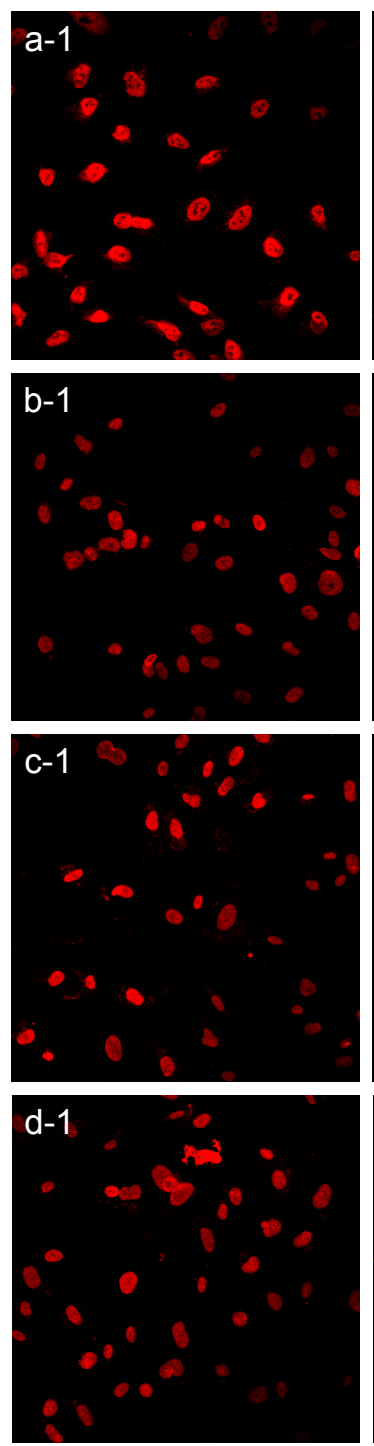
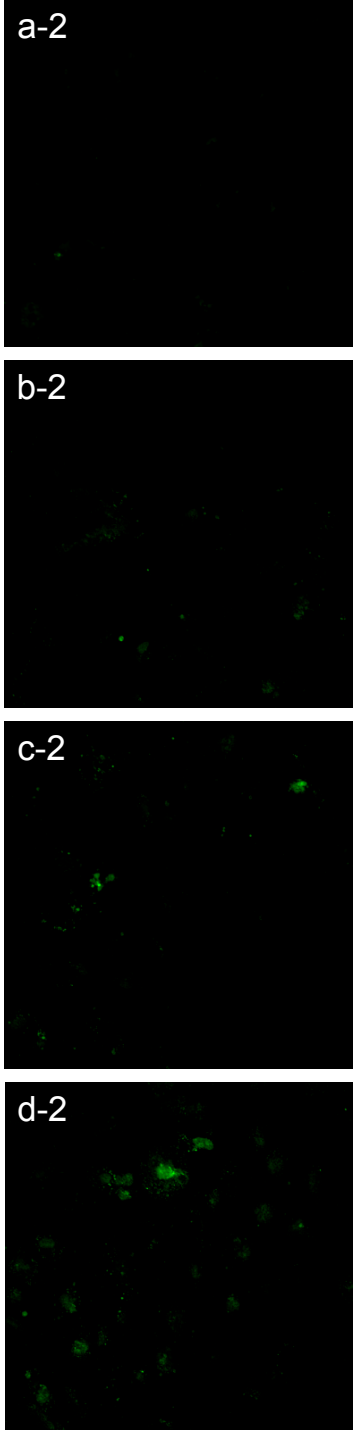
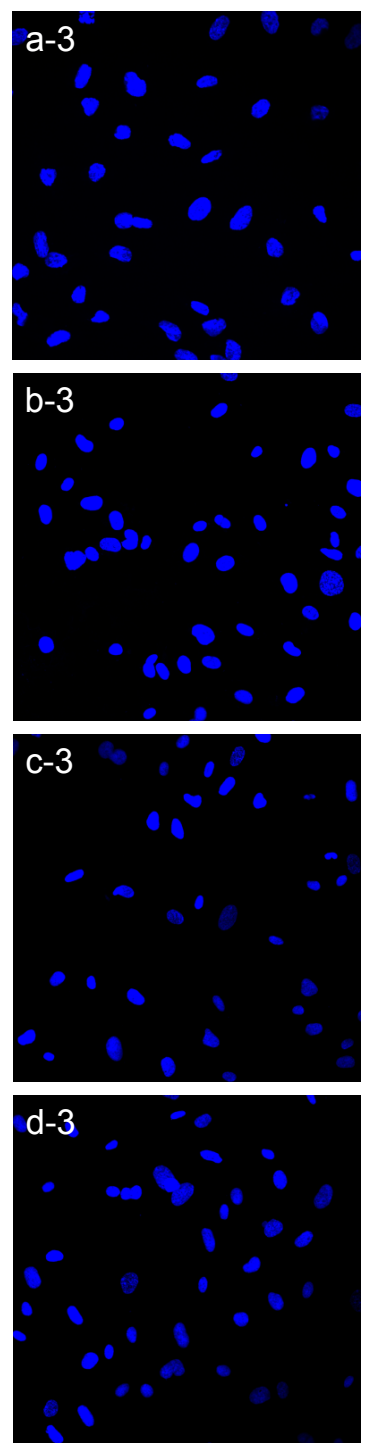
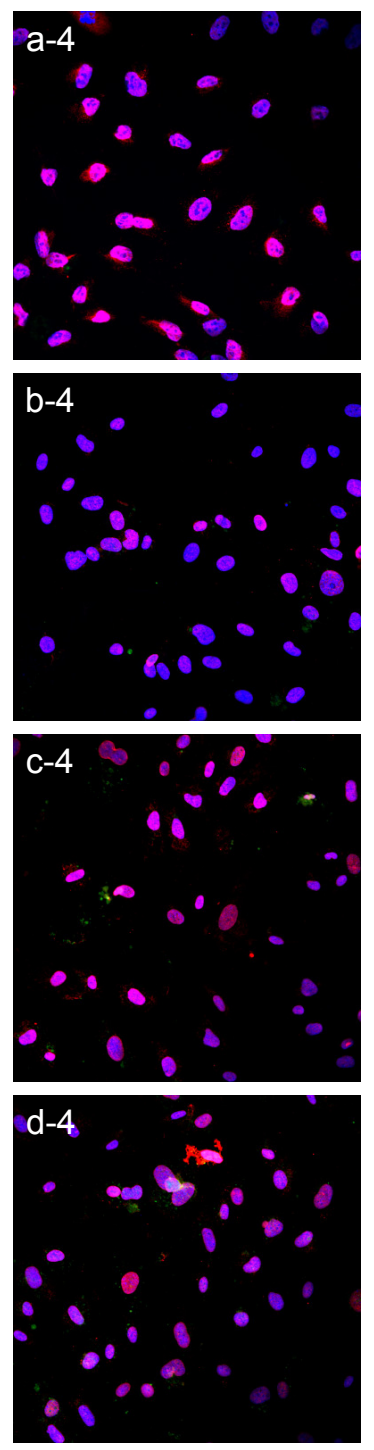

Figure 2 Delivery of RMP-7-Lf-QU-LS across the BBB $\left(r_{\mathrm{SA}}=5 \%\right)$.

Notes: (A) Viability of BBB cells (HBMECs and HAs; $C_{\mathrm{PMP} .7}=20 \mu \mathrm{g} / \mathrm{mL} ; C_{L}=20 \mu \mathrm{g} / \mathrm{mL} ; \mathrm{n}=3$ ); high viability of HBMECs and HAs suggested that RMP-7-Lf-QU-LS could not obviously imperil the BBB structure. (B) Effect of RMP-7 and Lf on TEER and ability of PI to cross the BBB (O, TEER of Lf-QU-LS; use $X$, and Y, axes; $\bullet$, TEER of RMP-7Lf-QU-LS; $C_{L f}=20 \mu \mathrm{g} / \mathrm{mL}$; use $X_{2}$ and $Y_{1}$ axes; - - -, control of TEER without carrier treatment; use $Y_{1}$ axis; $\mathrm{n}=3$; $\square$, ability of PI to cross the BBB with Lf-QU-LS; use $X_{1}$ and $Y_{2}$ axes; $\square$, ability of PI to cross the BBB with RMP-7-Lf-QU-LS; $C_{L f}=20 \mu \mathrm{g} / \mathrm{mL}$; use $X_{2}$ and $Y_{2}$ axes; -- -, control of ability of PI to cross the BBB without carrier treatment; use $Y_{2}$ axis; $n=3$ ); surface RMP-7 and Lf could slightly decrease the TEER and increase the ability of PI to cross the BBB. (C) Effect of RMP-7 and Lf on the ability of QU to cross the BBB (O, Lf-QU-LS; n=3;, RMP-7-Lf-QU-LS; $C_{\mathrm{Lf}}=20 \mu \mathrm{g} / \mathrm{mL} ; \mathrm{n}=3$ ); RMP-7-Lf-QU-LS could significantly increase the ability of QU to cross the BBB. (D) Fluorescent images of HBMECs-targeted RMP-7-Lf-QU-LS $\left(C_{\text {Lf }}=20 \mu \mathrm{g} / \mathrm{mL}\right)$ : (a) QU-LS, (b) Lf-QU-LS, (c) RMP-7-Lf-QU-LS $\left(C_{\text {RMP-7 }}=10 \mu g / m L\right)$, and $(d)$ RMP-7-Lf-QU-LS $\left(C_{\text {RMP-7 }}=20 \mu g / m L\right)$; red stains against LfR in (b) and against both $B_{2}$ bradykinin receptor and LfR in (a), (c), (d); green stains indicate carriers with FITC; blue stains indicate nuclei of HBMECs (DAPI); magnification 400x. RMP-7-Lf-QU-LS could target HBMECs and benefit the delivery of QU across the BBB. $r_{S A}$, mole percentage of SA in the lipid bilayer; $C_{\text {RMP-7 }}$, concentration of RMP-7; $C_{L}$, concentration of $L f ; P_{V, B B B}$, viability of BBB cells after treatment with RMP-7-Lf-QU-LS; $P_{B B B, P}$, ability of PI to permeate the BBB after treatment with RMP-7-Lf-QU-LS; $P_{\text {BBB,QU, }}$, ability of QU to cross the BBB using RMP-7-Lf-QU-LS.

Abbreviations: RMP-7-Lf-QU-LS, quercetin-encapsulated liposomes grafted with RMP-7 and lactoferrin; BBB, blood-brain barrier; HBMEC, human brain microvascular endothelial cell; HA, human astrocyte; Lf, lactoferrin; TEER, transendothelial electrical resistance; PI, propidium iodide; Lf-QU-LS, quercetin-liposomes grafted with lactoferrin; $\mathrm{QU}$, quercetin; QU-LS, quercetin-encapsulated liposomes; LfR, lactoferrin receptor; FITC, fluorescein isothiocyanate; DAPI, 4',6-diamidino-2-phenylindole; SA, stearylamine; LS, liposomes.

QU-LS could significantly retard the expressions of p-JNK, p-p38, and p-S202, and free QU led only to minor inhibition of their expressions. After treating with RMP-7-Lf-QU-LS, the levels of p-JNK, p-p38, and p-S202 in A $\beta$-insulted SK-N-MC cells were comparable to those of the control group. JNK belongs to the mitogen-activated protein kinase (MAPK) family. ${ }^{40}$ It was reported that JNK could be activated by $A \beta$, leading to tau protein phosphorylation. ${ }^{41}$ p38 also belongs to the MAPK family and is regarded as one of the key kinases in tau phosphorylation. ${ }^{42}$ Moreover, tau hyperphosphorylation induced by p-JNK and p-p38 is located at S202 in the early stage. ${ }^{43}$ It is well known that 
hyperphosphorylated tau can sequestrate soluble tau in the dysfunction of axonal transport in neurons and contributes to AD. Figure 3C shows the staining images of RMP-7-LfQU-LS adjacent to A $\beta$-insulted SK-N-MC cells. As seen in this figure, fluorescent red A $\beta$ surrounded SK-N-MC cells and attracted green RMP-7-Lf-QU-LS. Figure 3Ca and b shows that QU-LS and RMP-7-QU-LS appeared in the neighborhood of SK-N-MC cells due to recognition of A $\beta$. Compared with Figure $3 \mathrm{Cb}$, c, and d shows that surface Lf increased the quantity of green particles around SK-N-MC cells.

A

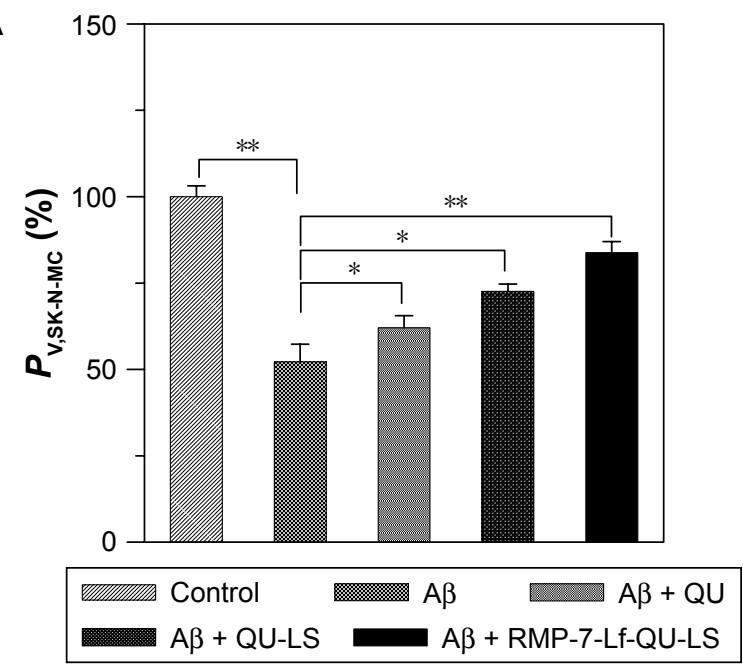

B a

C

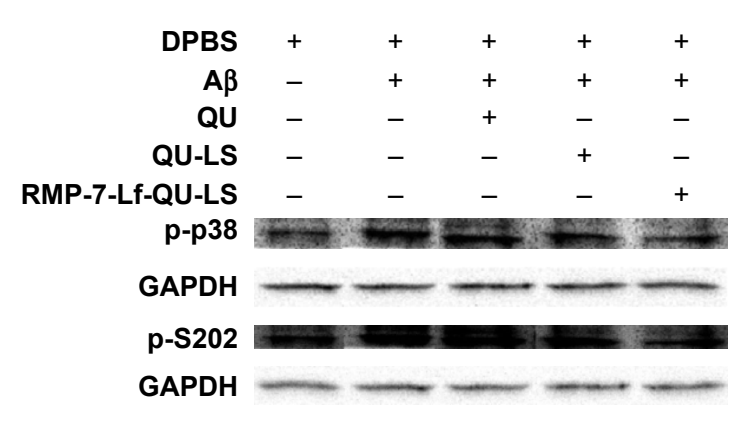

b
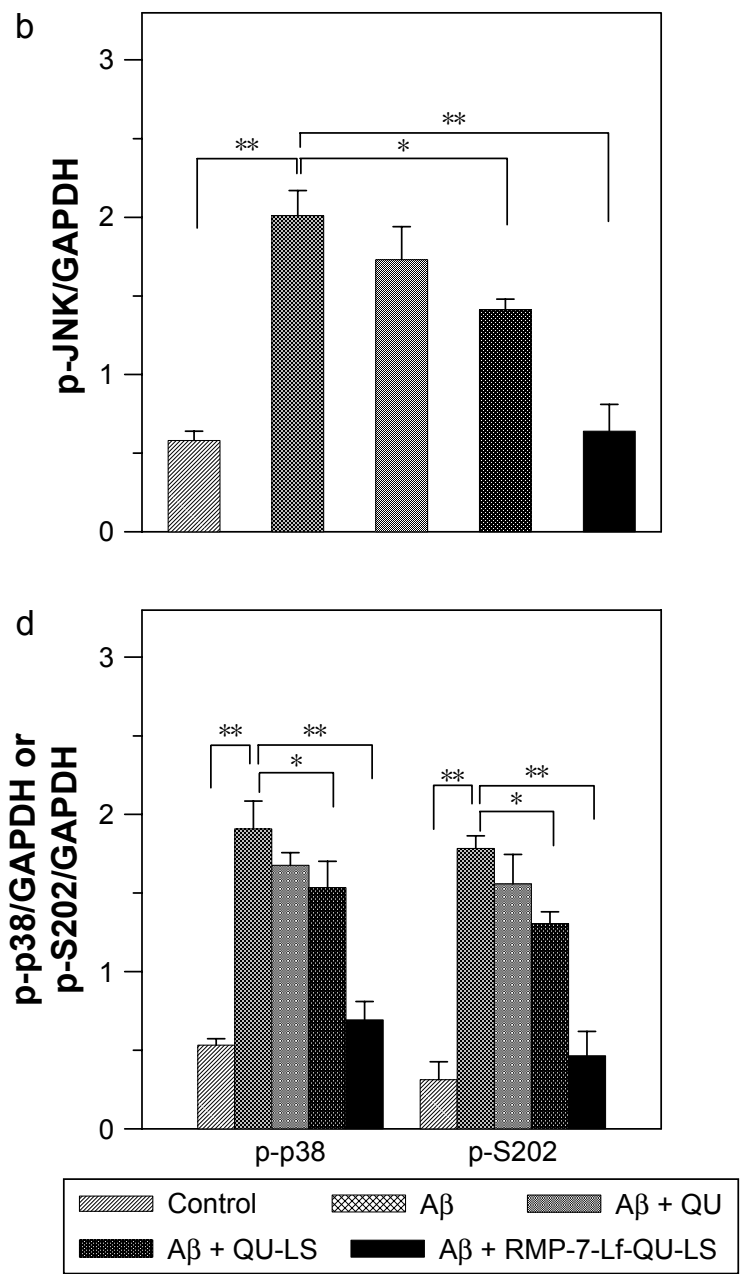

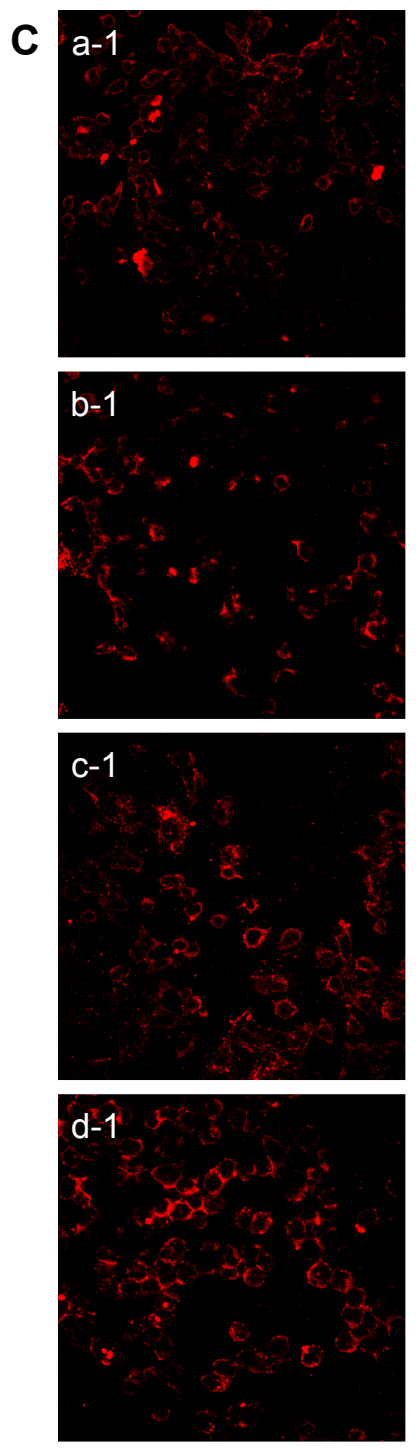
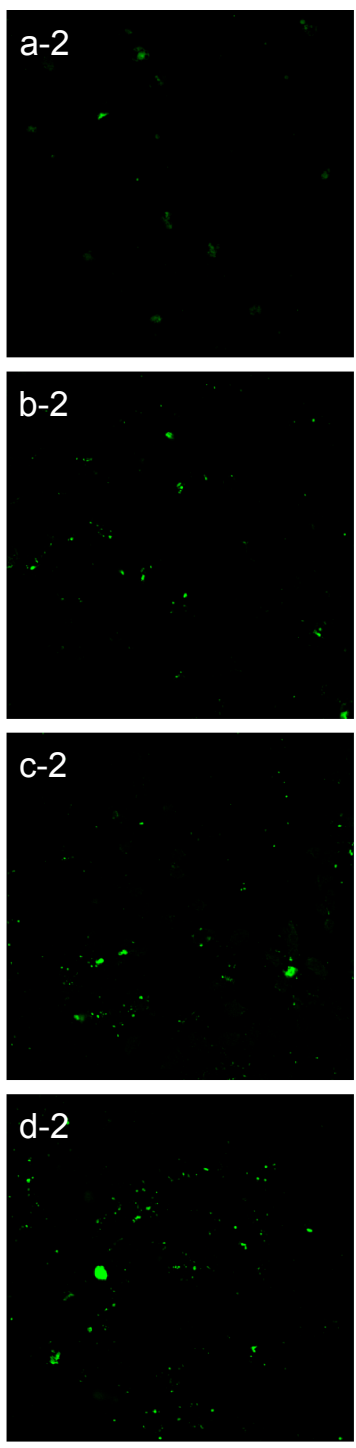
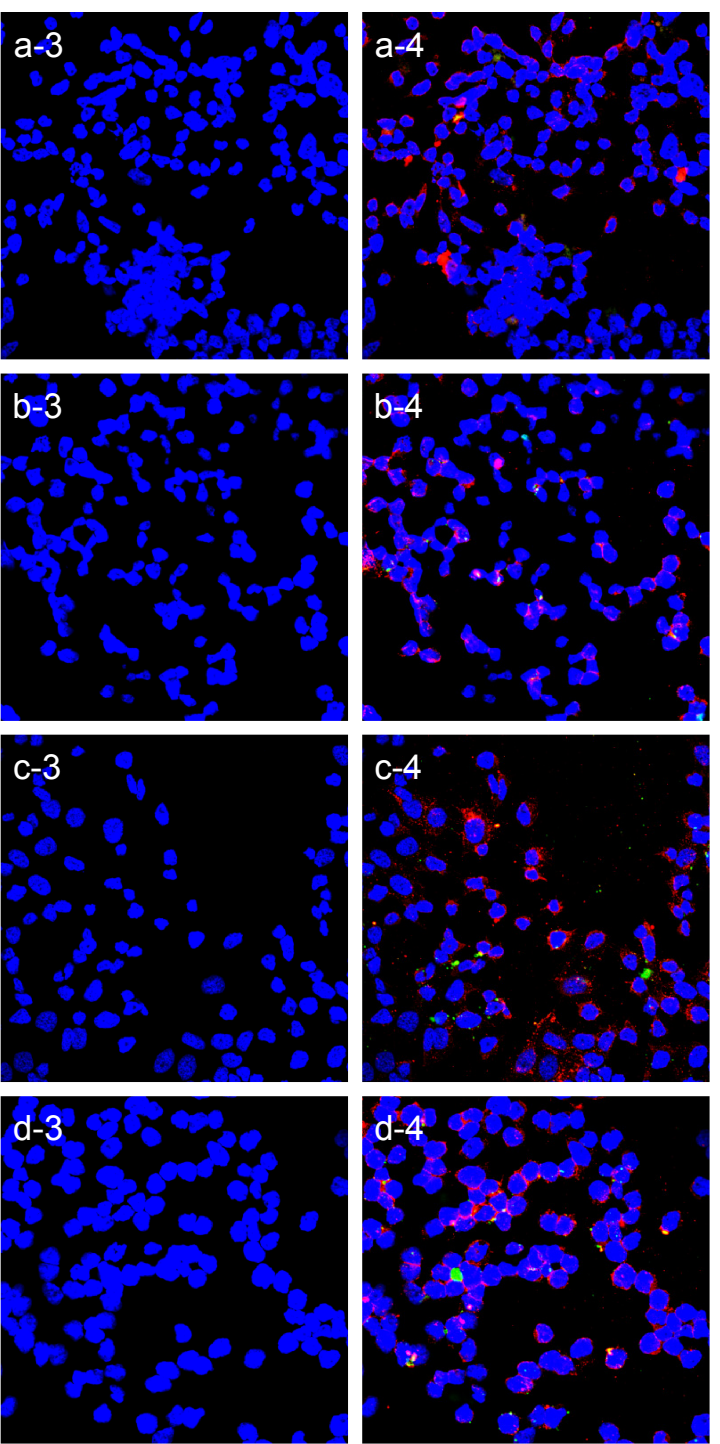

Figure 3 Treatment with RMP-7-Lf-QU-LS for SK-N-MC cells after insult with A $\beta\left(r_{\text {SA }}=5 \% ; C_{\text {RMP-7 }}=20 \mu g / \mathrm{mL}\right)$.

Notes: (A) Viability of SK-N-MC cells after treating with RMP-7-Lf-QU-LS $\left(C_{L f}=20 \mu \mathrm{g} / \mathrm{mL} ; * P<0.05 ; * * P<0.01\right.$; $\left.n=3\right)$; RMP-7-Lf-QU-LS could favor the rescue of degenerated SK-N-MC cells. (B) Protein level of P-JNK, P-p38, and p-S202 in SK-N-MC cells $\left(C_{\mathrm{Lf}}=20 \mu \mathrm{g} / \mathrm{mL}\right)$ : (a) and (c) Western blot, (b) protein ratio of $\mathrm{P}-\mathrm{JNK} / \mathrm{GAPDH}(* \mathrm{P}<0.05$; $* * P<0.01 ; n=3)$, and (d) protein ratio of $p-p 38 / G A P D H$ and $p-S 202 / G A P D H(* P<0.05 ; * * P<0.01 ; n=3)$; RMP-7-Lf-QU-LS could reduce the levels of $p-J N K, p-p 38$, and $p-S 202$ in Aß-insulted SK-N-MC cells to reduce apoptosis. (C) Fluorescent image of RMP-7-Lf-QU-LS around SK-N-MC cells: (a) QU-LS, (b) RMP-7-QU-LS, (c) RMP-7-Lf-QU-LS $\left(C_{\mathrm{Lf}}=10 \mu \mathrm{g} / \mathrm{mL}\right)$, and (d) RMP-7-Lf-QU-LS $\left(C_{\mathrm{Lf}}=20 \mu \mathrm{g} / \mathrm{mL}\right)$; red stains against $\mathrm{A} \beta$ antibody; green stains indicate carriers with FITC; blue stains indicate nuclei of SK-N-MC cells (DAPI); magnification 400×. RMP-7-Lf-QU-LS could recognize A $\beta$ around SK-N-MC cells and promote the efficacy of QU. $r_{\mathrm{SA}}$, mole percentage of SA in the lipid bilayer; $C_{\text {RMP-7 }}$, concentration of RMP-7; $C_{L f}$, concentration of Lf; $P_{\mathrm{V}, S K-N-M C}$, viability of SK-N-MC cells after treatment with RMP-7-Lf-QU-LS.

Abbreviations: RMP-7-Lf-QU-LS, quercetin-encapsulated liposomes grafted with RMP-7 and lactoferrin; A $\beta$, $\beta$-amyloid; p-JNK, phosphorylated c-Jun N terminal kinase; P-p38, phosphorylated p38; p-S202, phosphorylated tau protein at serine 202; GAPDH, glyceraldehyde 3-phosphate dehydrogenase; QU-LS, quercetin-encapsulated liposomes; RMP-7-QU-LS, quercetin-encapsulated liposomes grafted with RMP-7; FITC, fluorescein isothiocyanate; DAPI, 4',6-diamidino-2-phenylindole; QU, quercetin; SA, stearylamine; Lf, lactoferrin; DPBS, Dulbecco's phosphate-buffered saline.

Furthermore, an increase in the concentration of Lf enhanced the quantity of RMP-7-Lf-QU-LS near SK-N-MC cells. This was because RMP-7-Lf-QU-LS had an effect on targeting LfR and low-density lipoprotein receptor-related protein-1 (LRP1) on SK-N-MC cells. ${ }^{44}$ It has also been reported that LRP1 and its ligands exist in the $A \beta$ plaque of $A D$ patients and could form a complex to eliminate $A \beta{ }^{45,46}$ Thus, RMP7-Lf-QU-LS could conjugate $A \beta$ and be internalized by SK-N-MC cells, leading to a high uptake of QU and high likelihood of inhibiting neuronal apoptosis. Figure $3 \mathrm{C}$ is also consistent with Figure $3 \mathrm{~A}$ and B.

\section{Conclusion}

RMP-7-Lf-QU-LS was fabricated for targeting of the $\mathrm{BBB}$ and for delivering QU to SK-N-MC cells. Experimental evidence revealed that an increase in the mole percentage of SA increased the particle size and zeta potential but decreased the grafting efficiency of RMP-7 and Lf, in general. 
In addition, incorporated RMP-7 and Lf on the particles increased the level of surface nitrogen. RMP-7 and Lf also slightly enhanced the ability of PI to cross the BBB and reduced the TEER, indicating a minor tight junction opening after treatment with RMP-7-Lf-QU-LS. With regard to sustained release, dissolution of QU was in the order of QU-LS > Lf-QU-LS > RMP-7-Lf-QU-LS. Moreover, RMP7-Lf-QU-LS had low cytotoxicity to HBMECs and HAs and could be recognized by HBMECs for enhancing the transport of QU across the BBB. In terms of protein expression, $\mathrm{p}-\mathrm{JNK}$, p-p38, and p-S202 in SK-N-MC cells were in the order of free QU > QU-LS > RMP-7-Lf-QU-LS, suggesting that RMP-7-Lf-QU-LS could reduce neurotoxicity and improve neuronal survival. Hence, RMP-7-Lf-QU-LS can act as a potential neuroprotective system for inhibiting $A \beta$-induced apoptosis in $\mathrm{AD}$ management.

\section{Acknowledgment}

This work was supported by the Ministry of Science and Technology of the Republic of China (Taiwan) under grant number MOST 103-2221-E-194-043-MY3.

\section{Disclosure}

The authors report no conflicts of interest in this work.

\section{References}

1. Alzheimer's Association. 2016 Alzheimer's disease facts and figures. Alzheimers Dement. 2016;12(4):1-79.

2. Citron M. Alzheimer's disease: strategies for disease modification. Nat Rev Drug Dis. 2010;9(5):387-398.

3. Alzheimer A, Stelzmann RA, Schnitzlein HN, Murtagh FR. An English translation of Alzheimer's 1907 paper, "Uber eine eigenartige Erkankung der Hirnrinde”. Clin Anat. 1995;8(6):429-431.

4. Ballatore C, Lee VM, Trojanowski JQ. Tau-mediated neurodegeneration in Alzheimer's disease and related disorders. Nat Rev Neurosci. 2007;8(9):663-672.

5. Duyckaerts C, Delatour B, Potier MC. Classification and basic pathology of Alzheimer disease. Acta Neuropathol. 2009;118(1):5-36.

6. Biedler JL, Roffler-Tarlov S, Schachner M, Freedman LS. Multiple neurotransmitter synthesis by human neuroblastoma cell lines and clones. Cancer Res. 1978;38(11):3751-3757.

7. Nagele RG, D'Andrea MR, Anderson WJ, Wang HY. Intracellular accumulation of $\beta$-amyloid(1-42) in neurons is facilitated by the $\alpha 7$ nicotinic acetylcholine receptor in Alzheimer's disease. Neuroscience. 2002;110(2):199-211.

8. Ying G, Iribarren P, Zhou Y, et al. Humanin, a newly identified neuroprotective factor, uses the $\mathrm{G}$ protein-coupled formylpeptide receptorlike-1 as a functional receptor. J Immunol. 2004;172(11):7078-7085.

9. Bhutada P, Mundhada Y, Bansod K, et al. Ameliorative effect of quercetin on memory dysfunction in streptozotocin-induced diabetic rats. Neurobiol Learn Mem. 2010;94(3):293-302.

10. Choi GN, Kim JH, Kwak JH, et al. Effect of quercetin on learning and memory performance in ICR mice under neurotoxic trimethyltin exposure. Food Chem. 2012;132(2):1019-1024.

11. Kumar A, Sehgal N, Kumar P, Padi SS, Naidu PS. Protective effect of quercetin against ICV colchicine-induced cognitive dysfunctions and oxidative damage in rats. Phytother Res. 2008;22(12):1563-1569.
12. Yao Y, Han DD, Zhang T, Yang Z. Quercetin improves cognitive deficits in rats with chronic cerebral ischemia and inhibits voltage-dependent sodium channels in hippocampal CA1 pyramidal neurons. Phytother Res. 2010;24(1):136-140.

13. Manach C, Morand C, Crespy V, et al. Quercetin is recovered in human plasma as conjugated derivatives which retain antioxidant properties. FEBS Lett. 1998;426(3):331-336.

14. Bartus RT, Elliott PJ, Hayward NJ, Deen R, McEwen EL, Fisher SK. Permeability of the blood brain barrier by the bradykinin agonist, RMP-7: evidence for a sensitive, auto-regulated, receptor-mediated system. Immunopharmacology. 1996;33(1-3):270-278.

15. Emerich DF, Dean RL, Marsh J, et al. Intravenous Cereport (RMP-7) enhances delivery of hydrophilic chemotherapeutics and increases survival in rats with metastatic tumors in the brain. Pharm Res. 2000; 17(10):1212-1219.

16. Zhang X, Xie Y, Jin Y, et al. The effect of RMP-7 and its derivative on transporting Evens blue liposomes into the brain. Drug Deliv. 2004; 11(5):301-309.

17. Gonzalez-Chavez SA, Arevalo-Gallegos S, Rascon-Cruz Q. Lactoferrin: structure, function and applications. Int J Antimicrob Agents. 2009; 33(4):e301-e308.

18. Huang RQ, Ke WL, Qu YH, Zhu JH, Pei YY, Jiang C. Characterization of lactoferrin receptor in brain endothelial capillary cells and mouse brain. J Biomed Sci. 2007;14(1):121-128.

19. Kawamata T, Tooyama I, Yamada T, Walker DG, McGeer PL. Lactotransferrin immunocytochemistry in Alzheimer and normal human brain. Am J Pathol. 1993;142(5):1574-1585.

20. Kuo YC, Chen YC. Targeting delivery of etoposide to inhibit the growth of human glioblastoma multiforme using lactoferrin- and folic acidgrafted poly(lactide-co-glycolide) nanoparticles. Int J Pharm. 2015; 479(1):138-149.

21. Kuo YC, Chou PR. Neuroprotection against degeneration of SK-N-MC cells using neuron growth factor-encapsulated liposomes with surface cereport and transferrin. J Pharm Sci. 2014;103(8):2484-2497.

22. Ji B, Maeda A, Higuchi M, et al. Pharmacokinetics and brain uptake of lactoferrin in rats. Life Sci. 2006;78(8):851-855.

23. Kuo YC, Lu CH. Effect of human astrocytes on the characteristics of human brain-microvascular endothelial cells in the blood-brain barrier. Colloids Surf B Biointerfaces. 2011;86(1):225-231.

24. Kuo YC, Shih-Huang CY. Solid lipid nanoparticles with surface antibody for targeting the brain and inhibiting lymphatic phagocytosis. J Taiwan Inst Chem Eng. 2014;45(4):1154-1163.

25. Kuo YC, Liu YC. Cardiolipin-incorporated liposomes with surface CRM197 for enhancing neuronal survival against neurotoxicity. Int J Pharm. 2014;473(1-2):334-344.

26. Yousefi A, Esmaeili F, Rahimian S, Atyabi F, Dinarvand R. Preparation and in vitro evaluation of a pegylated nano-liposomal formulation containing docetaxel. Sci Pharm. 2009;77(2):453-464.

27. Nii T, Ishii F. Encapsulation efficiency of water-soluble and insoluble drugs in liposomes prepared by the microencapsulation vesicle method. J Agric Food Chem. 2005;298(1):198-205.

28. Wei M, Xu Y, Zou Q, et al. Hepatocellular carcinoma targeting effect of PEGylated liposomes modified with lactoferrin. Eur J Pharm Biopharm. 2012;46(3):131-141.

29. Antonio E, Khalil NM, Mainardes RM. Bovine serum albumin nanoparticles containing quercetin: characterization and antioxidant activity. J Nanosci Nanotechnol. 2016;16(2):1346-1353.

30. Cadena PG, Pereira MA, Cordeiro RBS, et al. Nanoencapsulation of quercetin and resveratrol into elastic liposomes. Biochim Biophys Acta. 2013;1828(2):309-316.

31. Shaji J, Iyer S. Preparation, optimization and in-vivo hepatoprotective evaluation of quercetin liposomes. Int J Curr Pharm Res. 2012; $4(2): 24-32$.

32. Lin CH, Al-Suwayeh SA, Hung CF, Chen CC, Fang JY. Camptothecinloaded liposomes with $\alpha$-melanocyte-stimulating hormone enhance cytotoxicity toward and cellular uptake by melanomas: an application of nanomedicine on natural product. J Tradit Complement Med. 2013; 3(2):102-109. 
33. Kuo YC, Lee CL. Methylmethacrylate-sulfopropylmethacrylate nanoparticles with surface RMP-7 for targeting delivery of antiretroviral drugs across the blood-brain barrier. Colloids Surf B. 2012;90: 75-82.

34. Xie Y, Ye L, Zhang X, et al. Transport of nerve growth factor encapsulated into liposomes across the blood-brain barrier: in vitro and in vivo studies. J Control Release. 2005;105(1-2):106-119.

35. Pardridge WM. Drug targeting to the brain. Pharm Res. 2007;24(9): 1733-1744.

36. Ying X, Wen H, Lua WL, et al. Dual-targeting daunorubicin liposomes improve the therapeutic efficacy of brain glioma in animals. J Control Release. 2010;141(2):183-192.

37. Gobbi M, Re F, Canovi M, et al. Lipid-based nanoparticles with high binding affinity for amyloid- $\beta_{1-42}$ peptide. Biomaterials. 2010;31(25): 6519-6529.

38. Shaykhalishahi H, Taghizadeh M, Yazdanparast R, Chang YT. Antiamyloidogenic effect of AA3E2 attenuates beta-amyloid induced toxicity in SK-N-MC cells. Chem Biol Interact. 2010;186(1):16-23.

39. Shimoji M, Imaizumi N, Aniya Y. Modulation of membrane-bound glutathione transferase activity by phospholipids including cardiolipin. Biol Pharm Bull. 2011;34(2):209-213.
40. Weston CR, Davis RJ. The JNK signal transduction pathway. Curr Opin Cell Biol. 2007;19(2):142-149.

41. Ploia C, Antoniou X, Sclip A, et al. JNK plays a key role in tau hyperphosphorylation in Alzheimer's disease models. J Alzheimers Dis. 2011; 26(2):315-329.

42. Churcher I. Tau therapeutic strategies for the treatment of Alzheimer's disease. Curr Top Med Chem. 2006;6(6):579-595.

43. Kuo YC, Lin CY, Li JS, Lou YI. Wheat germ agglutinin-conjugated liposomes incorporated with cardiolipin to improve neuronal survival for Alzheimer's disease treatment. Int J Nanomed. 2017;12:1757-1774.

44. Kuo YC, Wang CT. Protection of SK-N-MC cells against $\beta$-amyloid peptide-induced degeneration using neuron growth factor-loaded liposomes with surface lactoferrin. Biomaterials. 2014;35(22): 5954-5964.

45. Deane R, Sagare A, Zlokovic BV. The role of the cell surface LRP and soluble LRP in blood-brain barrier A $\beta$ clearance in Alzheimer's disease. Curr Pharm Design. 2008;14(16):1601-1605.

46. Zerbinatti CV, Wahrle SE, Kim H, et al. Apolipoprotein E and low density lipoprotein receptor-related protein facilitate intraneuronal A 342 accumulation in amyloid model mice. J Biol Chem. 2006; 281(47):36180-36186.
International Journal of Nanomedicine

\section{Publish your work in this journal}

The International Journal of Nanomedicine is an international, peerreviewed journal focusing on the application of nanotechnology in diagnostics, therapeutics, and drug delivery systems throughou the biomedical field. This journal is indexed on PubMed Central, MedLine, CAS, SciSearch $\AA$, Current Contents $₫ /$ Clinical Medicine,

\section{Dovepress}

Journal Citation Reports/Science Edition, EMBase, Scopus and the Elsevier Bibliographic databases. The manuscript management system is completely online and includes a very quick and fair peer-review system, which is all easy to use. Visit http://www.dovepress.com/ testimonials.php to read real quotes from published authors. 